\title{
Modifying the Cox maze procedure: Who should get a U?
}

\author{
Vinay Badhwar, MD
}

From the Department of Cardiothoracic Surgery, University of Pittsburgh, Pittsburgh, Pa.

Disclosures: Author has nothing to disclose with regard to commercial support.

Received for publication Nov 23, 2015; accepted for publication Nov 25, 2015; available ahead of print Jan 16, 2016.

Address for reprints: Vinay Badhwar, MD, Department of Cardiothoracic Surgery, University of Pittsburgh, 200 Lothrop St, C-724, Pittsburgh, PA 15213 (E-mail: badhwarv@upmc.edu).

J Thorac Cardiovasc Surg 2016;151:1070-2

$0022-5223 / \$ 36.00$

Copyright (c) 2016 by The American Association for Thoracic Surgery

http://dx.doi.org/10.1016/j.jtcvs.2015.11.051

In this issue of the Journal, Nitta and coauthors ${ }^{1}$ provide us with a retrospective analysis of their surgical ablation experience comparing 329 patients receiving a U lesion set (Cox maze IV procedure minus the left atrial [LA] superior box line between the right and left superior pulmonary veins) and 73 patients receiving a box lesion set (standard Cox maze IV procedure with a complete pulmonary vein-encircling lesion). Multivariable regression was applied between groups. In addition to the rhythm end point, 2-dimensional echocardiography informed a postoperative LA transport function end-point. This work is a testament to Dr Nitta's 15-year academic diligence. From birthing the concept of the radial procedure as a fellow at Washington University in the animal laboratory of Dr James Cox, ${ }^{2,3}$ then carefully maintaining a personal clinical series extrapolating the animal study model into what he now refers to as the U lesion set, Nitta and his team are to be congratulated on this exemplary focused pursuit of translational research.

In the original work by Nitta and coworkers ${ }^{3}$ in Cox's laboratory, 5 dogs underwent the radial procedure and 5 underwent the Cox maze procedure. After 6 weeks, the researchers found that atrial activation was delayed in the Cox maze group. In the current study of Nitta and coauthors, ${ }^{1}$ they refer to the $U$ lesion set as the radial lesion from this previous work. The central tenet of this longitudinal clinical study is that by eliminating the LA superior box line at the time of surgical ablation, patients may have improved LA transport function relative to those receiving a conventional box lesion set without impacting freedom from atrial fibrillation (AF). ${ }^{1}$ By not electrically isolating the posterior LA wall, the U lesion set theoretically permits activation and recruitment of this area between the pulmonary veins, perhaps contributing to LA contractile function.

To frame our interpretation of this interesting series, let us examine the electrophysiologic basis of the lesion sets, existing clinical experience, and the modality of LA transport functional assessment.

Cox demonstrated that once AF is induced, it can be characterized by the simultaneous existence of 2 or more

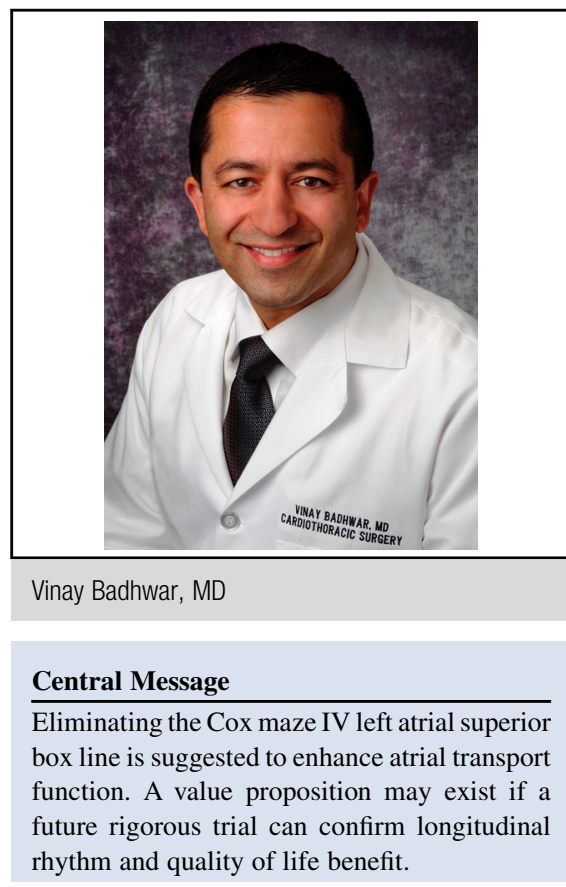

See Article page 1062 .

large macroreentrant atrial circuits. $^{2,4}$ The surgical ablation and rhythm restoration goals of the Cox maze procedure are to place lesions in the atria close enough together to prevent atrial macroreentry but permit sinus node impulse activation of both atria, the atrioventricular node, and the ventricles. As patients move from paroxysmal to nonparoxysmal AF with increasing chronicity, the underlying electrophysiologic mechanisms move from focal atrial or pulmonary vein triggers to self-perpetuating macroreentrant circuits secondary to areas of ultrastructural atrial fibrosis, often in the body, roof, and posterior wall of the LA. ${ }^{5-7}$ Although the current study of Nitta and coauthors ${ }^{1}$ did not demonstrate a significant difference in the rhythm end point at follow-up between $\mathrm{U}$ lesion set and box lesion set groups, both with an admirably high proportion of long-standing persistent cases, the $\mathrm{U}$ lesion set group had more cases of paroxysmal $\mathrm{AF}$ and the box lesion set group had more cases of persistent AF with atrial pathology. This may be a factor in the finding of increased LA transport function in the $U$ lesion set group relative to the box lesion set group from a limited assessment of the cases with evaluable echocardiograms $(177 / 402 ; 44 \%)$. Nitta and coauthors ${ }^{1}$ did note, however, that for some patients in whom complex fractionated electrograms could be identified posteriorly, they would 
recommend treatment with a box lesion set. Finally, by using delayed enhancement magnetic resonance imaging for contemporary assessments of patients with persistent $\mathrm{AF}$, others have identified atrial fibrosis and posterior foci of AF that may further question the prudence of deviating from the box lesion set in these patients. 8,9

Although the results of freedom from AF by Nitta and coauthors ${ }^{1}$ are indeed respectable, we must keep in mind that the commencement of this study predated existing Heart Rhythm Society recommendations for ablation follow-up. ${ }^{10}$ Although Nitta and coauthors ${ }^{1}$ diligently followed their patients up every 6 months, they dutifully disclose that their rhythm end point was informed most commonly by data from isolated electrocardiograms. Similarly, the current study of Nitta and coauthors ${ }^{1}$ also suffers from a lack of intergroup homogeneity in terms of AF type, concomitant operation, and energy source used. This may explain why other clinical experiences with partial or incomplete box lesion sets have not been as successful as those enjoyed by Nitta and coauthors. ${ }^{1}$ Damiano's group has suggested that it may be the incomplete box lesion set that is responsible for attrition of longitudinal rhythm success, particularly in patients with persistent AF. ${ }^{11-13}$

Finally, the LA transport end point for this study was derived from transthoracic echocardiography by means of peak early to late ventricular filling velocity (E/A) ratios. In normal physiologic states, LA mechanics span 3 distinct phases. ${ }^{14}$ The reservoir phase passively receives pulmonary venous return during left ventricular (LV) systole. The conduit phase permits passive emptying of the LA into the LV during early ventricular diastole. The contractile phase provides as much as an additional $25 \%$ of LV stroke volume in late ventricular diastole associated with atrial systolic contraction. In Nitta's original animal work $^{2}$ as well as in the current study of Nitta and coauthors, ${ }^{1}$ the use of pulse-wave Doppler was used to assess early and late ventricular filling velocities by measuring peak E/A ratios as a surrogate for documenting return of LA transport function. The use of $\mathrm{E} / \mathrm{A}$ ratios is quite reasonable to assess atrial contractile function in healthy patients with minimal atrial pathology. However, E/A ratios are often used to assess diastolic LV dysfunction and results are often affected by LV hypertrophy, aortic regurgitation, AF, pacing, tachycardia, and relative transmitral flow impairment that may be associated with a mitral repair or prosthetic replacement. Although mechanical valves were excluded from transmitral flow assessments in the current study of Nitta and coauthors, ${ }^{1}$ patients with mitral repair and bioprosthetic replacements as well as patients in AF were included and thus may confound interpretation of the LA transport function end point. Adjudication of E/A ratios and LA transport estimates are often based on a mathematical presumption of relative homogeneity of the LA. Human pathologic studies of patients in AF with mitral stenosis or regurgitation often suffer from mathematically nonlinear asymmetric dilatation, particularly between the right inferior pulmonary vein and the mitral annulus, further affecting the accuracy of echocardiographic estimation of LA transport by transmitral Doppler echocardiography. ${ }^{15}$ Other modalities, such as computed tomography and magnetic resonance imaging, have proved useful for a more anatomically derived assessment of atrial contractile function and may have use for future study.

Despite these limitations arising from the era of the study at its commencement, we thank Nitta and coauthors ${ }^{1}$ for their persistence and focus to follow their series and report their findings in this elegant contribution to the Journal. This work raises the following 2 questions:

1. Does the potential for improved LA transport translate into improvements in quality of life after surgical ablation?

2. If LA size and AF type were controlled for in a future matched analysis, would there be a difference between a $U$ lesion set and a box lesion set, and if so, for which AF types?

For now, any modification to the Cox maze procedure requires validation by a prospective trial with homogeneous AF type, energy source, and guideline-directed monitoring. Would the simplification of reducing a single lesion really save that much time? Obviously not, but if it can add improved atrial function and translate into improved outcome, then it may have a place in surgical decision making and resolve the real question arising from this work: Who should get a U?

\section{References}

1. Nitta T, Ishii Y, Fujii M, Miyagi Y, Sakamoto SI, Hiromoto A, et al. Restoration of sinus rhythm and atrial transport function after maze procedure: U lesion set versus box lesion set. J Thorac Cardiovasc Surg. 2016;151:1062-9.

2. Lawton JS, D'Amico TA. Historical perspectives of The American Association for Thoracic Surgery: James Lewis Cox (1942-). J Thorac Cardiovasc Surg. 2015; 149:1235-9.

3. Nitta T, Lee R, Watanabe H, Harris KM, Erikson JM, Schuessler RB, et al Radial approach: a new concept in surgical treatment for atrial fibrillation. II. Electrophysiologic effects and atrial contribution to ventricular filling. Ann Thorac Surg. 1999;67:36-50.

4. Cox JL, Canavan TE, Schuessler RB, Cain ME, Lindsay BD, Stone C, et al. The surgical treatment of atrial fibrillation. II. Intraoperative electrophysiologic mapping and description of the electrophysiologic basis of atrial flutter and atrial fibrillation. J Thorac Cardiovasc Surg. 1991;101:406-26.

5. Burstein B, Nattel S. Atrial fibrosis: mechanisms and clinical relevance in atrial fibrillation. J Am Coll Cardiol. 2008;51:802-9.

6. Allessie M, Ausma J, Schotten U. Electrical, contractile and structural remodeling during atrial fibrillation. Cardiovasc Res. 2002;54:230-46.

7. Kazui T, Henn MC, Watanabe Y, Kovács SJ, Lawrance CP, Greenberg JW, et al. The impact of 6 weeks of atrial fibrillation on left atrial and ventricular structure and function. J Thorac Cardiovasc Surg. 2015;150:1602-8.e1.

8. Marrouche NF, Wilber D, Hindricks G, Jais P, Akoum N, Marchlinski F, et al Association of atrial tissue fibrosis identified by delayed enhancement MRI and atrial fibrillation catheter ablation: the DECAAF study. JAMA. 2014;311: 498-506. Erratum in: JAMA. 2014;312:1805. 
9. Jadidi AS, Cochet H, Shah AJ, Kim SJ, Duncan E, Miyazaki S, et al. Inverse relationship between fractionated electrograms and atrial fibrosis in persistent atrial fibrillation: combined magnetic resonance imaging and high-density mapping. J Am Coll Cardiol. 2013;62:802-12.

10. Calkins H, Kuck KH, Cappato R, Brugada J, Camm AJ, Chen SA, et al. 2012 HRS/EHRA/ECAS expert consensus statement on catheter and surgical ablation of atrial fibrillation: recommendations for patient selection, procedural techniques, patient management and follow-up, definitions, endpoints, and research trial design. J Interv Card Electrophysiol. 2012;33:171-257.

11. Damiano RJ Jr, Schwartz FH, Bailey MS, Maniar HS, Munfakh NA, Moon MR, et al. The Cox maze IV procedure: predictors of late recurrence. J Thorac Cardiovasc Surg. 2011;141:113-21.
12. Weimar T, Bailey MS, Watanabe Y, Marin D, Maniar HS, Schuessler RB, et al. The Cox-maze IV procedure for lone atrial fibrillation: a single center experience in 100 consecutive patients. J Interv Card Electrophysiol. 2011; 31:47-54.

13. Gillinov M, McCarthy PM, Benussi S, Damiano RJ Jr, Ad N. Surgical ablation for atrial fibrillation. Semin Thorac Cardiovasc Surg. 2015;27:65-79.

14. Mehrzad R, Rajab M, Spodick DH. The three integrated phases of left atrial macrophysiology and their interactions. Int J Mol Sci. 2014;15:15146-60.

15. Corradi D, Callegari S, Benussi S, Maestri R, Pastori P, Nascimbene S, et al. Myocyte changes and their left atrial distribution in patients with chronic atrial fibrillation related to mitral valve disease. Hum Pathol. 2005;36: 1080-9. 